zenem $\mathrm{LiNO}_{3}$ als in festem $\mathrm{Li}_{2} \mathrm{SO}_{4}$. Eine weitere Diskussion dieser Tatsache soll anstehen, bis die laufenden Messungen an anderen Salzschmelzen abgeschlossen sind.
Die Arbeit ist von Wilhelm och Martina Lundgrens vetenskapsfond und Statens råd för atomforskning finanziell unterstützt worden. Wir danken Herrn M. Lövenby für seine Mitwirkung bei der massenspektrometrischen Analyse.

\title{
Improvement of Simple Wave Functions in Given Regions of Molecular Systems
}

\author{
By T. Szondy \\ Research Group for Theoretical Physics of the Hungarian Academy of Sciences, Budapest \\ (Z. Naturforschg. 17 a, 552-553 [1962] ; eingegangen am 14. April 1962)
}

\begin{abstract}
Es wird eine Näherungsmethode für die Bestimmung der Wellenfunktion molekularer Systeme vorgeschlagen, von welcher man in erster Linie in denjenigen Gebieten des Konfigurationsraumes eine gute Näherung erwarten kann, wo eine beliebige, gegebene Gewichtsfunktion große Absolutwerte hat.
\end{abstract}

The approximate wave functions of molecular systems are usually determined by the variational principle

$$
\langle H\rangle \equiv\langle\psi|H| \psi\rangle=\text { extr.; }\langle\psi \mid \psi\rangle=1
$$

where $H$ denotes the Hamiltonian of the system. In domains which are of little importance for the value of $\langle H\rangle$, these wave functions may be a very poor approximation of the exact one even if $\langle H\rangle$ is a good approximation of the empirical energy of the system. Thus, if these domains are important for the expectation value of a physical quantity $f$, $\langle\psi|f| \psi\rangle$ may be a very poor approximation of the exact expectation value ${ }^{1}$.

Since the improvement of the approximation by increasing the number of the variational parameters in $\psi$ leads to rather tedious calculations and the convergence is often unsatisfactory, Preuss ${ }^{2}$ raised the idea to determine the optimal values of the parameters from such criteria which ensure a good approximation of the exact wave function primarily in those regions where $|f|$ is large in order to obtain good results with relatively simple wave functions ${ }^{3}$. This can be achieved by determining the

1 In the following we assume that $f$ is only a function of the co-ordinates of the electrons but not a function of their momenta.

2 H. Preuss, Z. Naturforschg. 13 a, 439 [1958] ; 16 a, 598 [1961].

3 Thus the optimal values of the parameters slightly depend on the physical quantity $f$, the expectation value of which we want to calculate. approximate wave function from the Preuss equation ${ }^{4}$

$$
\int|f(H-\langle H\rangle) \psi|^{2} \mathrm{~d} \tau=\min ; \quad\langle\psi \mid \psi\rangle=1 .
$$

In the following a new method will be proposed for the determination of $\psi$, from which one can similarly expect an improved approximation in domains where $|f|$ is large, but which avoids the calculation of matrix elements involving $H^{2}$ and therefore in some cases can be considerably less tedious than the Preuss method.

Let be $\psi$ an approximate wave function involving the free parameters $\alpha_{1} \ldots \alpha_{m}$. We choose a set of linearly independent functions $f_{1} \ldots f_{n} ;(n \gg m)$, which depend on the same co-ordinates as $f$, and which satisfy the conditions

$$
\begin{gathered}
\frac{1}{n} \sum_{i=1}^{n} f_{i} \approx f, \\
\left|f-f_{i}\right| \ll|f|,
\end{gathered}
$$

at least in those domains which are important for the value of $\langle\psi|f| \psi\rangle$. $n$ can be even infinite, e. g. if the $f_{i}$ 's involve continuously varying parameters.

\footnotetext{
4 There are two reasonable choices of $\langle H\rangle$ : (a) to determine $\langle H\rangle$ by eq. (1), (b) to vary $\psi$ also in $\langle H\rangle$. Naturally $\psi$ must be flexible enuogh to make possible a good approximation of the exact $\langle H\rangle$. Choice (b) slightly increases the sensitiveness of the method in regions where $|f|$ is small and this may be advantageous in cases when such regions occupy a considerable part of the system.
} 
Let us consider the expression ${ }^{4-6}$

$$
\left.W \equiv \frac{1}{n} \sum_{i=1}^{n} \frac{\left\langle\psi\left|f_{i}(H-\langle H\rangle)\right| \psi\right\rangle}{\left\langle\psi\left|f_{i}\right| \psi\right\rangle}\right|^{2} ; \quad\langle\psi \mid \psi\rangle=1 .
$$

$W$ will be small (a) if $\psi$ is a good approximation of the exact wave function, primarily in those domains where $|f|$ is large (b) if the positive and negative parts of the integrands in each

$$
\left\langle\psi\left|f_{i}(H-\langle H\rangle)\right| \psi\right\rangle
$$

compensate each other. The possibility (b) can be excluded by the careful choice of the $f_{i}$ ' $\mathrm{s}^{7}$. Thus, if the values of the parameters $\alpha_{1} \ldots \alpha_{m}$ are determined by the condition

$$
W=\min
$$

we can expect that $\psi$ will be a good approximation of the exact wave function, primarily in those regions, where $|f|$ is large.

There is undoubtedly some arbitrariness in the choice of the $f_{i}$ 's, similar to the arbitrariness in the choice of the basic points in the "local energy method" 8 . However, if $\psi$ is a good approximation of the exact wave function, the best values of the parameters $\alpha_{1} \ldots \alpha_{m}$ are rather insensitive to changes in the $f_{i}$ 's which are compatible with the conditions (3), and thus the result is only slightly affected by this arbitrariness.

This can readily be seen in the following example. The expectation value of $f=r_{1}{ }^{2}$ has been calculated in the ground state of the helium atom by means of the wave function $\psi \sim \exp \left\{-Z\left(r_{1}+r_{2}\right)\right\}$, regarding $Z$ as a variable parameter. Four different sets of $f_{i}$ 's have been used:

1. $f_{1}=f ; \quad(n=1)$

2. $f_{1}=f ; f_{2}=r_{1}^{2} \exp \left\{-\frac{1}{10} Z r_{1}\right\} ; f_{3}=r_{1}^{2} \exp \left\{\frac{1}{10} Z r_{r_{1}}\right\}$; $(n=3)$

3. $f_{i}=r_{1}^{2} \exp \left\{\beta_{i} r_{1}\right\}, \beta_{i}$ varying continuously in the

5 If the $f_{i}$ 's involve continuously varying parameters, the summation over $i$ involves also an integration over these parameters.

6 We assume that $\left\langle\psi\left|f_{i}\right| \psi\right\rangle$ is of the same order of magnitude for every $f_{i}$. If $f_{i} \geqq 0$ these conditions are automatically satisfied because of the conditions (3). If $\langle\psi|f| \psi\rangle \approx 0$ it is advisable to replace $f$ by $f^{2}$.

7 If $\psi$ is only an approximate wave function, $W=0$ presumes $\left\langle\psi\left|f_{i}(H-\langle H\rangle)\right| \psi\right\rangle=0$ for all $f_{i}$ 's, and there are only $m$ parameters to satisfy these $n \gg m$ equations. interval $-\frac{1}{10} Z \leqq \beta_{i} \leqq \frac{1}{10} Z ; \quad(n=\infty)$. The integration over $\beta_{i}$ has been carried out by the Simpson rule.

4. $f_{1}=f ; \quad f_{2}=r_{1}^{2} \exp \left\{-\frac{1}{10} Z_{r_{1}}\right\} ; \quad(n=2)$.

The last set of $f_{i}$ 's is slightly incompatible with the condition (3 a)!

In Table 1 the values obtained for $\langle\psi|f| \psi\rangle^{9}$ are compared with the exact value ${ }^{10}$ and with the value obtained by the usual variational method.

\begin{tabular}{|l|c|c|}
\hline \multirow{2}{*}{} & $\langle\psi|f| \psi\rangle$ & Best $Z$ \\
\cline { 2 - 3 } & \multicolumn{2}{|c|}{ (atomic } \\
\hline Exact & 1,193 & - \\
Variational & 1,053 & 1,688 \\
$1)$ & 1,136 & 1,625 \\
$2)$ & 1,147 & 1,617 \\
$3)$ & 1,139 & 1,623 \\
$4)$ & 1,145 & 1,619 \\
\hline
\end{tabular}

Table 1.

Not e. If we replace $\langle H\rangle$ in $\left\langle\psi\left|f_{i}(H-\langle H\rangle)\right| \psi\right\rangle$ by the empirical value, a considerable part of the integration difficulties can be avoided by suitable choice of the $f_{i}$ 's. A possible choice of this kind is

$$
f_{i}=\frac{1}{\psi} \prod_{k} x_{k}{ }^{p_{i k}} y_{k}{ }^{q_{i k}} z_{k}^{r_{i k}}
$$

or any linear combination of such products, where $x_{k}, y_{k}, z_{k}$ are the cartesian co-ordinates of the $k$ 'th electron and $p_{i k}, q_{i k}, r_{i k}$ are non-negative integers satisfying the condition $\left\langle\psi\left|f_{i}\right| \psi\right\rangle \neq 0$. If $\psi$ is an LCAO-MO wave function which may involve configuration interaction, the most difficult integrals occuring in $\left\langle\psi\left|f_{i}(H-\langle H\rangle)\right| \psi\right\rangle$ are two-centre Coulomb integrals. This procedure may be useful even if it requires loosening of the restrictions (3).

Acknowledgment. Thanks are due to Prof. P. Gombás, Dr. E. Kapuy and in particular to Dr. H. Preuss for valuable remarks.

8 The local energy method is closely related to the method proposed in this paper. If the $f_{i}$ 's are Dirac $\delta$-functions and each member of the sum is multiplied by some weight factor, eq. (5) reduces to the basic equation of the local energy method.

9 The results listed in Table 1 were calculated by choice (b) of foot-note ${ }^{4}$. Choice (a) gave only very slightly different results.

10 C. L. Pekeris, Phys. Rev. 115, 1216 [1959]. 